\title{
Médiévales
}

Langues, Textes, Histoire

79 | automne 2020

Éthiopie, Nubie, Égypte

\section{Vida Alice Tyrrell, Merovingian Letters and Letter Writers}

Turnhout, Brepols (« Publications of the Journal of Medieval Latin », 12), 2019, xxxi-386 p.

\section{Martin Gravel}

\section{OpenEdition}

\section{Journals}

Édition électronique

URL : https://journals.openedition.org/medievales/11647

DOI : $10.4000 /$ medievales. 11647

ISSN : 1777-5892

Éditeur

Presses universitaires de Vincennes

\section{Édition imprimée}

Date de publication : 28 décembre 2020

Pagination : 240-242

ISBN : 978-2-37924-146-8

ISSN : 0751-2708

\section{Référence électronique}

Martin Gravel, «Vida Alice Tyrrell, Merovingian Letters and Letter Writers », Médiévales [En ligne], 79 |

automne 2020, mis en ligne le 28 janvier 2021, consulté le 07 janvier 2023. URL : http://

journals.openedition.org/medievales/11647 ; DOI : https://doi.org/10.4000/medievales.11647

Ce document a été généré automatiquement le 7 janvier 2023.

Tous droits réservés 


\section{Vida Alice Tyrrell, Merovingian Letters and Letter Writers}

Turnhout, Brepols (« Publications of the Journal of Medieval Latin », 12), 2019, xxxi-386 p.

\section{Martin Gravel}

\section{RÉFÉRENCE}

Vida Alice Tyrrell, Merovingian Letters and Letter Writers, Turnhout, Brepols

(« Publications of the Journal of Medieval Latin », 12), 2019, xxxI-386 p.

1 Vida Alice Tyrrell publie une version remaniée de sa thèse de doctorat soutenue en 2012 à l'Université de Toronto. Il faut se féliciter de ce nouvel ajout à la série de travaux qui ont donné un grand élan à l'étude de l'épistolaire médiéval, tout au long de la décennie 2010, d'autant plus que, si le corpus des lettres de la Gaule mérovingienne n'est pas le plus riche quantitativement, ce n'est pas non plus celui qui profite de la plus grande attention.

2 L'ouvrage se présente à la fois comme un outil de travail et une série d'études dialoguant entre elles. Il ne s'agit donc pas d'une thèse au sens strict du terme ; il n'y a pas ici de démonstration englobante, mais bien un assemblage. Cette approche est opportune, dans la mesure où le corpus d'environ six cents lettres est polarisé autour de certaines collections, de certains auteurs, alors que la période d'étude couvre près de trois siècles. Ainsi, à défaut d'une grande idée, ce livre en propose plusieurs de moindre portée, mais souvent stimulantes, en plus d'un nouvel outillage.

Les trois premiers chapitres procèdent de façon chronologique. V. A. Tyrrell parcourt le corpus qu'elle a assemblé en partant du cercle des grands épistoliers gaulois de la fin du $v^{e}$ siècle (Sidoine Apollinaire, Ruricius de Limoges, Avit de Vienne) pour cheminer jusqu'à la correspondance de Boniface et Lull au milieu du viII ${ }^{\mathrm{e}}$ siècle. Sur son parcours, elle prend soin de relever toutes les lettres qui ont été écrites ou reçues en Gaule, ce qui l'amène à récupérer des pièces de collections non gauloises, dans les Variae de 
Cassiodore, dans le registre de Grégoire le Grand ou les lettres wisigothiques... Elle ajoute des textes épistolaires insérés dans d'autres sources, hagiographiques notamment. Toutes ces lettres étaient déjà connues et éditées, mais le fait de les réunir et de les disposer dans le temps pour en faire l'étude permet de les saisir dans leur ensemble et dans leurs transformations, ce qui est une première contribution de l'ouvrage.

Les quatre derniers chapitres se distinguent les uns des autres, chacun portant sur un thème particulier: d'abord les lettres des rois et des papes (chap.4), puis la correspondance des femmes (chap. 5), le rôle des porteurs et des cadeaux (chap. 6), les citations scripturaires (chap. 7). Ce sont quatre études séparées, mais l'auteure leur donne une même tonalité, leur assure des échanges, et donc une forme de cohérence. La table des matières donne à croire que ce livre fonctionne comme un menu, où chacun trouverait son bonheur, mais l'ensemble se lit très bien du début à la fin. Cette entreprise permet de saisir d'un même coup d'œil tout le panorama épistolaire mérovingien. De ce point de vue, il est regrettable que l'auteure n'ait pas développé sa conclusion générale, qui lui offrait pourtant la possibilité de révéler ce panorama et de partager ses impressions à l'échelle de son sujet (p. 219-220).

Du côté de l'outillage complémentaire, le lecteur trouvera une présentation succincte des principales collections épistolaires traitées (p. XXII-XXXI), puis une liste des citations scripturaires repérées dans les lettres (p. 221-225). Mais la plus importante contribution se trouve dans les fiches descriptives consacrées à chacune des lettres du corpus, lesquelles occupent une importante partie de l'ouvrage (p. 227-356). Chaque lettre est présentée dans un résumé de quelques lignes, permettant de survoler l'ensemble ou de le fouiller au gré des curiosités, ce qui offre de véritables perspectives de travail. En lui-même, cet appendice suffit à assurer l'utilité durable de cette publication, en particulier pour l'enseignement. Il est complété par un index des noms de personne qui renvoie aux lettres.

6 La nature composite de l'ouvrage empêche cependant de le saisir en bloc: le lecteur y trouvera beaucoup de bonnes idées, de belles analyses, mais aussi quelques propositions plus difficiles à suivre, le tout sur une variété de thèmes particulièrement étendue. Cet aspect composite s'affirme davantage par l'insertion de plusieurs excursus sur différents thèmes, lesquels méritent parfois une dizaine de pages, notamment : le carmen figuratum de Venance Fortunat (p.42-49), l'affrontement épistolaire de Chrodebert et Importun (p.66-81), l'influence de Julien Pomère sur une lettre de Boniface (p. 85-89). Il y en a d'autres et toutes devraient être annoncées dans la table des matières. Il en découle que la mosaïque que propose ce livre se ne prête pas bien à une appréciation d'un seul tenant. Surtout, elle appelle des réactions variées, d'un lecteur à l'autre. La suite de ce compte rendu est à l'avenant: ses observations ne peuvent être que discutables; d'aucuns n'y verront peut-être que des détails.

7 Parmi les propositions stimulantes qui pimentent les études de V. A. Tyrrell, nous en retiendrons quatre, pour servir d'illustrations de ce qu'elles proposent de meilleur. La première contribue à la cohérence de la partie chronologique de l'ouvrage (chap. 1-3) : elle décrit la tension qui se maintient tout au long de la période entre l'art délicatement entortillé de l'amitié épistolaire tardo-antique et la rigueur d'expression associée à l'ascétisme de Césaire d'Arles et Julien Pomère. Dans ce contexte, l'auteure repère dans une lettre de Boniface la première citation épistolaire de la Vita contemplativa (p. 85-89). La deuxième proposition montre, par l'étude de toutes les traces laissées par les lettres 
de Childebert $\mathrm{I}^{\text {er }}(\dagger 558)$, qu'il est possible d'évaluer l'étendue véritable d'une correspondance, même s'il ne reste en fait qu'une seule lettre envoyée au nom de ce roi (p. 94-98). Dans un champ de sources aussi mal servi par les aléas de la conservation, la recherche doit s'emparer des moindres indices, et c'est ce que parvient à faire l'auteure dans ce cas. Troisièmement, suivant Thomas F. X. Noble, elle contribue à replacer les lettres pontificales au centre des échanges de l'Europe latine, au-delà du pontificat de Grégoire le Grand (p. 106 passim). Cela n'est pas négligeable, dans la mesure où, trop souvent, l'autorité du pape est conçue à tort comme une invention post-carolingienne. Cette autorité a une histoire, certes, mais elle ne commence pas au $\mathrm{IX}^{\mathrm{e}}$ siècle, et encore moins au Xle. Enfin, l'auteure insiste à juste titre pour comparer les lettres les plus finement composées à des gestes d'amitié s'apparentant à des cadeaux, à des objets littéraires. Cela est sans doute évident pour quiconque a fréquenté les vers de Venance Fortunat, mais si c'est une évidence, nous ne prenons pas la juste mesure de sa portée. Il n'est pas facile aujourd'hui, à l'ère du mail, du post et du SMS, de concevoir qu'un texte puisse être l'objet d'une longue fréquentation, proche de celle que l'on réserve pour les icônes. V. A. Tyrrell nous aide à le comprendre.

Le livre offre plusieurs autres belles pépites, mais on y croise aussi des idées discutables. Mentionnons justement la comparaison récurrente entre la lettre d'amitié tardo-antique ou médiévale et les échanges rendus possibles par les médias sociaux aujourd'hui (par exemple p. 41-42, 91). Au-delà d'une similarité d'intention superficielle - créer du lien -, la comparaison ne tient pas et il faut même dire qu'elle trompe. La lettre d'amitié est faite pour être apprivoisée et fréquentée, peut-être toute la vie ; en termes d'effet médiatique, cela n'a rien à voir avec l'instantanéité électronique et le babil des médias sociaux. La lettre d'amitié ne peut pas nous être révélée par cette comparaison qui occulte le fait qu'elle nous est devenue étrange, difficile à saisir, notamment dans la préciosité de son expression, ses possibilités hypnotiques, son ouverture à l'imagination. Les lettres du haut Moyen Âge sont le contraire des gestes frénétiques de la communication électronique, elles ne les annoncent pas et elles ne les remplacent pas.

9 Ensuite, V. A. Tyrrell propose une étude tout à fait intéressante des lettres de femmes, dont il reste vingt textes et de nombreuses mentions. Ces vingt lettres forment un ensemble inestimable pour la recherche, mais il faut se garder d'en presser des statistiques, sous prétexte qu'il n'y a rien d'autre à mettre sous la loupe. À ce titre, la comparaison stylistique des douze lettres "continentales» aux huit lettres «insulaires » fait s'élever un mirage. On ne saura jamais si ses volutes sont dues aux hasards de la conservation, car, après tout, les huit lettres insulaires sont toutes de la collection de Boniface et Lull, donc du milieu du viII siècle. Le même problème se pose pour l'étude des lettres « émotives ». Il est possible que ces lettres aient été conservées parce qu'elles semblaient alors représentatives d'une forme d'écriture émotive "féminine », il est tout aussi possible que cette sélection cache le proverbial iceberg des lettres qui ne participaient pas de ce genre de clichés. Ces lettres méritent toute l'attention que leur consacre V. A. Tyrrell, mais la seule approche méthodologiquement concevable reste l'étude pièce par pièce: elles ne forment pas un ensemble représentatif, ou plutôt, si elles forment un ensemble représentatif, nous ne le saurons jamais.

10 Enfin, si l'ouvrage a un défaut d'ensemble, il se trouve dans la relative pauvreté des références dans les notes de bas de page et dans la bibliographie secondaire, qui fait à 
peine douze pages. Certes, les nouveaux défis de l'édition forcent à réduire le plus possible cette forme de contribution scientifique. Dans le cas d'une thèse stricto sensu, cela n'est pas excessivement dommageable, mais dans le cas d'un ouvrage dont la principale contribution tient de la synthèse, de l'introduction au corpus et de la constitution d'un outillage, cela ne se justifie pas. Certes, il y a ici plusieurs objets d'étude réunis, ce qui rend la tâche particulièrement ardue. Il est vrai que la bibliographie a été augmentée depuis 2012, mais elle reste courte. C'est d'autant plus dommage que, manifestement, l'auteure connaît très bien les principales langues de la recherche et, bien sûr, le latin "mérovingien ». Parions qu'elle a été, comme plusieurs jeunes collègues, victime de l'injonction productiviste - une thèse en trois ans!? - qui prive la recherche des vitamines et des minéraux dont elle aura toujours besoin pour vivre. Des exemples? Elle s'occupe de la question des transformations de la langue sans trop prêter attention aux travaux de Michel Banniard, qui est pourtant la référence ${ }^{1}$. Les soutiens historiographiques du chapitre sur les messagers et les cadeaux sont aussi trop peu nombreux.

11 Mais une bibliographie indigente ne saurait ruiner un bon livre, et assurément la thèse publiée de V. A. Tyrrell est un très bon livre, appelé à servir d'outil et d'inspiration sur la durée. Il complétera le guide Late Antique Letter Collections publié en 2017, dont la chronologie s'arrête au début $\mathrm{du} \mathrm{vI}^{\mathrm{e}}$ siècle. Grâce à ces travaux, l'expression du desideratum historiographique pour les lettres du haut Moyen Âge commence à ressembler à un cliché dont il faudrait se débarrasser.

\section{NOTES}

1. Il y a bien d'autres absents, ni négligeables, ni trop récents. Par exemple, autour de Didier de Cahors : R. LE JAN, « Le lien social entre Antiquité et haut Moyen Âge : l'amitié dans les collections de lettres gauloises ", dans D. HÄGERMANN, W. HAUBRICHS, J. JARNÜT éd., Akkulturation. Probleme einer germanisch-romanischen Kultursynthese in Spätantike und frühem Mittelalter, Berlin, 2004, p. 528-546; B. SCHMEIDLER, «Über Briefsammlungen des früheren Mittelalters in Deutschland und ihre kritische Verwertung », Vetenskaps-societeten i Lund: Årsbok. Yearbook of the New Society of Letters at Lund, 1926, p. 5-27 ; S. LINGER, «L'écrit à l'époque mérovingienne d'après la correspondance de Didier, évêque de Cahors (630-655) », Studi Medievali, 3e série, 33 (1992), p. 799-823.

\section{AUTEURS}

\section{MARTIN GRAVEL}

Université Paris 8 Vincennes-Saint-Denis, Arscan-Thémam 